\title{
TNF and IL-6 differentially regulate the production of DKK-1, a master regulator of bone remodelling, by fibroblast-like synoviocytes
}

\author{
N Yeremenko ${ }^{1 *}$, R Bisoendial ${ }^{1}$, J Zwerina ${ }^{2}$, G Schett ${ }^{2}$, P P Tak ${ }^{3}$, D Baeten ${ }^{3}$ \\ From 5th European Workshop on Immune-Mediated Inflammatory Diseases \\ Sitges-Barcelona, Spain. 1-3 December 2010
}

\begin{abstract}
Background
Different inflammatory joint diseases have distinct patterns of bone damage with pronounced erosions in rheumatoid arthritis (RA), a combination of bone destruction and formation in psoriatic arthritis (PsA), and new bone formation in spondyloarthritis (SpA). Although the underlying mechanisms remain elusive, blocking of DKK-1 reverses the bone-destructive pattern to a boneforming pattern in experimental arthritis.
\end{abstract}

\section{Aim}

In order to delineate the role of DKK-1 in arthritis, we analyzed the regulation of DKK-1 expression in the inflamed peripheral joint of different types of inflammatory arthritis ex-vivo and in fibroblast-like synoviocyte (FLS) cultures in vitro.

\section{Patients and methods}

IL-6, DKK-1, TNF, and IL-1 beta levels in synovial fluid (SF) and synovial FLS lines were determined by ELISA. DKK-1 serum levels were assessed before and after IL-6R blockade in RA patients.

\section{Results}

SF DKK-1 levels were similar between 4 disease groups with a striking variability within each cohort. As DKK-1 production is strongly upregulated by TNF, we explored this inter-individual variability by correlating DKK-1 levels with pro-inflammatory cytokines levels. TNF and IL-1 beta levels were significantly higher in RA than SpA SF and did not correlate with DKK-1. In contrast, there was a striking inverse correlation between DKK-1 and IL-6 in both RA and SpA. Consistent with these data, in vitro DKK-1 production by FLS was strongly induced by TNF but clearly suppressed by IL-6 in dosedependent manner. Preliminary data suggest that this regulation of DKK-1 by IL- 6 is also relevant in vivo as treatment with the anti-IL-6 R antibody, tocilizumab, induced a transient upregulation of DKK-1 serum levels in RA patients.

\section{Conclusions}

DKK-1 is abundantly expressed in the inflamed joint of both destructive and remodelling forms of arthritis. However, DKK-1 production by FLS is differentially regulated by TNF and IL-6. The relative balance between these factors in the arthritic joints may determine the pattern of inflammation-induced tissue remodelling.

\section{Author details}

${ }^{1}$ Clinical Immunology and Rheumatology, Academic Medical Center/ University of Amsterdam, Amsterdam, The Netherlands. ${ }^{2}$ Internal Medicine 3, Friedrich-Alexander University of Erlangen-Nuremberg, Germany. ${ }^{3}$ Division of Clinical Immunology and Rheumatology, Academic Medical Centre/ University of Amsterdam, The Netherlands.

Published: 25 November 2010

\section{doi:10.1186/1479-5876-8-S1-P43}

Cite this article as: Yeremenko et al:: TNF and IL-6 differentially regulate the production of DKK-1, a master regulator of bone remodelling, by fibroblast-like synoviocytes. Journal of Translational Medicine 2010 8(Suppl 1):P43. 